\title{
Research Article \\ On the Multiplicity of a Proportionally Modular Numerical Semigroup
}

\author{
$\mathrm{Ze} \mathrm{Gu}$ \\ School of Mathematics and Statistics, Zhaoqing University, Zhaoqing, Guangdong 526061, China \\ Correspondence should be addressed to Ze Gu; guze528@sina.com
}

Received 10 June 2021; Accepted 20 August 2021; Published 8 September 2021

Academic Editor: Alfred Peris

Copyright (c) $2021 \mathrm{Ze} \mathrm{Gu}$. This is an open access article distributed under the Creative Commons Attribution License, which permits unrestricted use, distribution, and reproduction in any medium, provided the original work is properly cited.

A proportionally modular numerical semigroup is the set $S(a, b, c)$ of nonnegative integer solutions to a Diophantine inequality of the form $a x \bmod b \leq c x$, where $a, b$, and $c$ are positive integers. A formula for the multiplicity of $S(a, b, c)$, that is, $m(S(a, b, c))=$ $\lceil k b / a\rceil$ for some positive integer $k$, is given by A. Moscariello. In this paper, we give a new proof of the formula and determine a better bound for $k$. Furthermore, we obtain $k=1$ for various cases and a formula for the number of the triples $(a, b, c)$ such that $k \neq 1$ when the number $a-c$ is fixed.

\section{Introduction and Preliminaries}

Given two integers $m$ and $n$ with $n \neq 0$, we denote by $m \bmod n$ the remainder of the division of $m$ by $n$. A proportionally modular Diophantine inequality is an expression of the form $a x \bmod b \leq c x$, where $a, b$, and $c$ are positive integers. In $[1,2]$, it is shown that the set $S(a, b, c)$ of nonnegative integer solutions of the former inequality is a numerical semigroup; that is, it is a subset of the set $\mathbb{N}$ of the nonnegative integers that is closed under addition, contains 0 , and whose complement in $\mathbb{N}$ is finite. This type of numerical semigroups is known as proportionally modular numerical semigroups. If $S$ is a numerical semigroup and $d$ is a positive integer, then we denote by $S / d$ the set $\{x \in \mathbb{N} \mid \mathrm{d} x \in S\}$. It is clear that $S / d$ is a numerical semigroup containing $S$. We say that $S / d$ is the quotient of $S$ by $d$. Given two relatively prime positive integers $a_{1}$ and $a_{2}$, we denote by $\left\langle a_{1}, a_{2}\right\rangle$ the numerical semigroup generated by them, that is, $\left\langle a_{1}, a_{2}\right\rangle=\left\{s_{1} a_{1}+s_{2} a_{2} \mid s_{1}, s_{2} \in \mathbb{N}\right\}$. From Theorem 6.18 in [1], we know that $\left\langle a_{1}, a_{2}\right\rangle / d$ is a proportionally modular numerical semigroup, and every proportionally modular numerical semigroup can be represented in this form. Let $S$ be a numerical semigroup. We say that $S$ has a Toms decomposition if there exist $q_{1}, \ldots, q_{n}, m_{1}, \ldots, m_{n}$ and $L$ such that (1) $\operatorname{gcd}\left(q_{i}, m_{i}\right)=\operatorname{gcd}\left(L, q_{i}\right)=\operatorname{gcd}\left(L, m_{i}\right)=1$ for all $i \in\{1, \ldots, n\}$ and (2) $S=(1 / L) \cap_{i=1}^{n}\left\langle q_{i}, m_{i}\right\rangle$. Let $a, b$, and $c$ be positive integers. We say that the numerical semigroup $\langle a, b\rangle / c$ is a Toms block if $\operatorname{gcd}(a, b)=\operatorname{gcd}(a, c)=\operatorname{gcd}(b, c)=1$. Observe that $(1 / L) \cap_{i=1}^{n}\left\langle q_{i}, m_{i}\right\rangle=\cap_{i=1}^{n}\left(\left\langle q_{i}, m_{i}\right\rangle / L\right)$. So, a numerical semigroup admits a Toms decomposition if and only if it can be expressed as an intersection of finitely many Toms blocks with the same denominator. In $[1,3]$, it is shown that numerical semigroups having a Toms decomposition are precisely those numerical semigroups that can be expressed as a finite intersection of proportionally modular numerical semigroups.

As usual, for a rational number $r,\lceil r\rceil$ denotes the least integer not smaller than $r$ and $\lfloor r\rfloor$ denotes the greatest integer not bigger than $r$. If $S$ is a numerical semigroup, then the least positive integer that belongs to $S$ is an important invariant of $S$ called the multiplicity of $S$, and we denote it by $m(S)$ (see, for example, [1]). Some partial results on the invariant of a proportionally modular numerical semigroup 
are given in $[4,5]$. In particular, a formula, that is, $m(S(a, b, c))=\lceil k b / a\rceil$ where $k$ is some positive integer, is given by Moscariello in [6].

The contents of this paper are organized as follows. In Section 2, we give a clearer proof on the formula given in [6] and the bound of $k$. In Section 3, we obtain $k=1$ for various cases and a formula for the number of the triples $(a, b, c)$ such that $k \neq 1$ when the number $a-c$ is fixed. In Section 4 , we describe an algorithm that allows us to calculate the multiplicity of $S(a, b, c)$ and apply the algorithm to some questions.

\section{A Formula for $m(S(a, b, c))$}

As the inequality $a x \bmod b \leq c x$ has the same set of integer solutions as $(a \bmod b) x \bmod b \leq c x$, we do not lose generality by supposing that $a<b$. If $c \geq a$, then $S(a, b, c)=\mathbb{N}$; thus, we may also suppose that $c<a$. In this paper, unless otherwise stated, we suppose that $a, b$, and $c$ are positive integers satisfying $c<a<b$.

Lemma 1 (Proposition 1 [7]). $S(a, b, c)=S(b+c-a, b, c)$.

The following lemma has as a consequence that we may also suppose that $a \leq(b+c) / 2$.

\section{Remark 1}

(1) Under the above notations, we have $m(S(a, b, c))<b$. In fact, for any positive integer $x$, if $c x \geq b-1$, then $x \in S(a, b, c)$. It follows that $m(S(a, b, c)) \leq(b-1) / c \leq b-1<b$.

(2) Let $a$ and $b$ be positive integers such that $a<b$. Then, for each integer $k$ there exists exactly one integer $\alpha_{k}$ such that $k b \leq a \alpha_{k}<k b+a$. Moreover, $\alpha_{k}=\lceil k b / a\rceil$. Indeed, since $0 \leq(-k b) \bmod a<a$, we have $k b \leq k b+(-k b) \bmod a<k b+a$. Therefore, $k b \leq a\lceil k b / a\rceil<k b+a$. Uniqueness follows from the fact that the set $\{k b, k b+1, \ldots, k b+a-1\}$ consists of a consecutive integers and therefore contains exactly one multiple of $a$.

As a consequence of the uniqueness of $\alpha_{k}$ in Remark 1 (2), we obtain the following result.

Lemma 2. Let $a$ and $b$ be positive integers such that $a<b$, and let $k$ be an integer. Then, $a\lceil k b / a\rceil$ is the least multiple of $a$ in the set $\{k b, k b+1, \ldots, k b+b-1\}$.

Remark 2. For an integer $x$, if $k b \leq a x<(k+1) b$, then $\lfloor a x / b\rfloor=k$. Thus, $a x \bmod b=a x-k b$.

Now, we are ready to prove the result given in [6] concerning the form of the multiplicity of $S(a, b, c)$.

Theorem 1 (Proposition 3 in [6]). Under the above hypothesis and notations, we have $m(S(a, b, c)) \in$ $\{\lceil k b / a\rceil \mid k \in\{1,2, \ldots, a-1\}\}$.
Proof. Let $m=m(S(a, b, c))$, then $m<b$ by Remark 1 (1). Thus, there exists $k \in\{0,1, \ldots, a-1\}$ such that $k b \leq a m<(k+1) b$, i.e., $m \geq\lceil k b / a\rceil$. If $m>\lceil k b / a\rceil$, then $m-1 \geq\lceil k b / a\rceil$, and we have $k b \leq a(m-1)<(k+1) b$. By Remark 2, we have $a(m-1) \bmod b=a(m-1)-$ $k b=a m-k b-a=a m \bmod b-a$. Since $m \in S(a, b, c)$, we have $a m \bmod b \leq c m$. Thus $a(m-1) \bmod b=a m \bmod b$ $-a \leq c m \quad-a<c m-c=c(m-1) . \quad$ Consequently, $m-1 \in S(a, b, c)$. From the fact that $m$ is the multiplicity of $S(a, b, c)$, we have $m-1=0$ and so $m=1$. Thus, $a=a \bmod b \leq c$ which contradicts that $c<a$. Hence, $m=\lceil k b / a\rceil$.

Finally, if $k=0$, then $m=\lceil 0 b / a\rceil=0$ which contradicts the definition of $m$. Therefore, $k$ must not be 0 , and this completes the proof.

From the preceding theorem, it follows that $m(S(a, b, c))=\lceil\xi b / a\rceil$, $\xi=\min \{k \in\{1,2, \ldots, a-1\} \mid[k b / a] \in S(a, b, c)\}$. The following three lemmas will allow us to reformulate this fact.

Lemma 3. Let $k \in\{1,2, \ldots, a-1\}$. Then, $\lceil k b / a\rceil \in S(a, b, c)$ if and only if $(-k b) \bmod a \leq k b c /(a-c)$.

Proof. Our conclusion can be obtained from the following deduction:

$$
\begin{aligned}
&\left\lceil\frac{k b}{a}\right\rceil \in S(a, b, c) \Leftrightarrow a\left\lceil\frac{k b}{a}\right\rceil \bmod b \leq c\left\lceil\frac{k b}{a}\right\rceil, \\
& \Leftrightarrow a\left\lceil\frac{k b}{a}\right\rceil \bmod b=a\left\lceil\frac{k b}{a}\right\rceil-k b \leq c\left\lceil\frac{k b}{a}\right\rceil \\
& \Leftrightarrow(a-c)\left\lceil\frac{k b}{a}\right\rceil \leq k b \\
& \Leftrightarrow(a-c) \frac{k b+(-k b) \bmod a}{a} \leq k b \\
& \Leftrightarrow(-k b) \bmod a \leq \frac{k b c}{a-c} .
\end{aligned}
$$

Lemma 4. Under the above notations, we have $\xi \leq\lfloor a(1-(1 / b)) / c\rfloor$.

Proof. According to Remark 1 (1), we know that $m(S(a, b, c)) \leq(b-1) / c$. It follows that $\lceil\xi b / a\rceil \leq(b-1) / c$ and so $(\xi b / a) \leq(b-1) / c$. Thus, $\xi \leq(a(1-(1 / b)) / c)$. Since $\xi$ is a positive integer, we have $\xi \leq\lfloor a(1-(1 / b)) / c\rfloor$.

Lemma 5. $\lfloor a(1-(1 / b)) / c\rfloor \leq a-1$, where the equality holds if and only if $c=1$.

Proof. Observing that $\lfloor a(1-(1 / b)) / c\rfloor \leq a(1-(1 / b)) / c<a$, we have $\lfloor a(1-(1 / b)) / c\rfloor \leq a-1$. If $c=1$, then $\lfloor a(1-(1 /$ $b)) / c\rfloor=\lfloor a(1-(1 / b))\rfloor=\lfloor a-(a / b)\rfloor=a-1$. Conversely, if the equality holds, $(a-1) \leq(a(1-(1 / b)) / c)$. Thus, $c \leq(a-(a / b)) /(a-1)=1+(b-a) /(b(a-1))$. Since $c$ is a 
positive integer, we have $c \leq\lfloor 1+(b-a) /(b(a-1))\rfloor=1$ and so $c=1$. follows.

Therefore, we can reformulate the fact in Theorem 1 as

Corollary 1. Under the above notations, we have $m(S(a, b, c))=\lceil\xi b / a\rceil$, where $\xi=\min \{k \in\{1,2, \ldots,\lfloor a(1-$ $(1 / b)) / c\rfloor\} \mid(-k b) \bmod a \leq k b c /(a-c)\}$.

\section{The Cases of $m(S(a, b, c))==\lceil b / a\rceil$ and $m(S(a, b, c)) \neq\lceil b / a\rceil$}

In this section, we study the cases of $k=1$ and the number of the triples $(a, b, c)$ such that $k \neq 1$ if the number $a-c$ is fixed.

As a consequence of Corollary 1 and the proof of Lemma 3 , we have the following result.

Theorem 2. $m(S(a, b, c))=\lceil b / a\rceil$ if and only if $(-b) \bmod a \leq b c /(a-c)$ if and only if $\lceil b / a\rceil \leq b /(a-c)$.

Corollary 2. If $a, b$, and $c$ satisfy one of the following conditions:

(1) $a-c \leq(a b /(a+b-1))$,

(2) $a-c \leq c+1$,

then $m(S(a, b, c))=\lceil b / a\rceil$.

Proof

(1) If $a-c \leq a b /(a+b-1)$, then $b c \geq(a-1)(a-c)$. Thus, $b c /(a-c) \geq a-1 \geq(-b) \bmod a$. By Theorem 2 , we have $m(S(a, b, c))=\lceil b / a\rceil$.

(2) If $a-c \leq c+1$, then $b c /(a-c) \geq b c /(c+1)$. Since $a<b$, we have $b \geq a+1$. Thus, $b c /(c+1) \geq((a+1) c /$ $(c+1))=a-(a-c) /(c+1) \geq a-1 \geq(-b) \bmod a$. It follows from Theorem 2 that $m(S(a, b, c))$ $=\lceil b / a\rceil$.

Remark 3. Since $c$ is a positive integer, $c+1 \geq 2$. If $a-c=1$ or 2 , then $m(S(a, b, c))=\lceil b / a\rceil$.

\section{Example 1}

(1) Let $a=13, b=70, \quad$ and $c=4$. Then, $S(a, b, c)=\{0,6,7,11,12,13,14,15\} \cup\{x$

$\in \mathbb{N} \mid x \geq 17\}$. As $\quad(a-c)(a+b-1)$ $=9 \times 82=738 \leq 910=13 \times 70=a b$, it follows that $m(S(a, b, c))=\lceil b / a\rceil=\lceil 70 / 13\rceil=6$.

(2) Let $\quad(a, b, c)=(5,42,2)$. Then, $S(a, b, c)=\{0,9,10,11,12,13,14\} \cup\{x \in \mathbb{N} \mid x \geq 17\}$. Since $a-c=3=c+1$, we have that $m(S(a, b, c))=\lceil b / a\rceil=\lceil 42 / 5\rceil=9$.

Theorem 3. Let $a-c=d$. Then, the number $N_{d}$ of the triples $(a, b, c)$ such that $m(S(a, b, c)) \neq\lceil b / a\rceil$ is given by

$$
N_{d}=\frac{1}{2} \sum_{i=1}^{d-2}\left\lfloor\frac{d-1}{i}\right\rfloor[d-1-i+(d-1) \bmod i] .
$$

Proof. If $a-c=d$, then $a \geq d+1$. By Corollary 2 (2), if $m(S(a, b, c)) \neq\lceil b / a\rceil$, then $a<2 d-1$. Thus, $a \in\{d+1$, $d+2, \ldots, 2 d-2\}$. Let $a=d+i$ (i.e., $c=i$ ) for some $i \in\{1,2, \ldots, d-2\}$ and $n_{i}$ be the number of $b$ such that $m(S(a, b, c)) \neq\lceil b / a\rceil$. By Theorem 2 , we know that $m(S(d+$ $i, b, i))=\lceil b / a\rceil$ if and only if $\lceil b /(d+i)\rceil \leq b / d$, that is, $m(S(d+i, b, i)) \neq\lceil b / a\rceil$ if and only if $j(d+i)<b<(j+1) d$ for some positive integer $j$ (notice that $b>d+i$ ). For a positive integer $j$, we obtain the number $d-1-i j$ of $b$ satisfying this condition between $j(d+i)$ and $(j+1) d$. Since $d-1-i j$ is a natural number, we have $j \leq\lfloor(d-1) / i\rfloor$. Thus, $n_{i}=\sum_{j=1}^{\lfloor(d-1) / i\rfloor}(d-1-i j)$. Therefore,

$$
\begin{aligned}
N_{d} & =\sum_{i=1}^{d-2} n_{i}, \\
& =\sum_{i=1}^{d-2} \sum_{j=1}^{\lfloor(d-1) / i\rfloor}(d-1-i j) \\
& =(d-1) \sum_{i=1}^{d-2}\left\lfloor\frac{d-1}{i}\right\rfloor-\sum_{i=1}^{d-2}\left\lfloor\frac{(d-1) / i\rfloor}{j=1} i j\right. \\
& =(d-1) \sum_{i=1}^{d-2}\left\lfloor\frac{d-1}{i}\right\rfloor-\sum_{i=1}^{d-2} i \frac{\lfloor(d-1) / i\rfloor(\lfloor(d-1) / i\rfloor+1)}{2} \\
& =\sum_{i=1}^{d-2}\left\lfloor\frac{d-1}{i}\right\rfloor\left[d-1-\frac{1}{2} i\left(\left\lfloor\frac{d-1}{i}\right\rfloor+1\right)\right] \\
& =\frac{1}{2} \sum_{i=1}^{d-2}\left\lfloor\frac{d-1}{i}\right\rfloor[d-1-i+(d-1) \bmod i] .
\end{aligned}
$$

This completes our proof.

Remark 4. By the formula in Theorem 3, we obtain that $N_{3}=1$ and the triple $(a, b, a-3)$ satisfying $m(S(a, b, a-$ $3)) \neq\lceil b / a\rceil$ is exactly $(4,5,1)$. Moreover, $N_{4}=4, N_{5}=9, N_{6}=17$, and $N_{7}=27$. This gives the integer sequence $A 078567$ (see [8]) $1,4,9,17,27,41,57,77, \ldots$. In fact, we know from [8] that $a_{n}=\sum_{i=1}^{n-1} \sum_{j=1}^{\lfloor(n-1) / i]}(n-i j)$ is one of the formulations of the integer sequence $A 078567$. If $i \mid n, \quad$ then $\lfloor n / i\rfloor=n / i=\lfloor(n-1) / i\rfloor+1 \quad$ and $n-i\lfloor n / i\rfloor=n-i(n / i)=0$. Otherwise, $\lfloor n / i\rfloor=\lfloor(n-1) / i\rfloor$. Thus, $a_{n}=\sum_{i=1}^{n-1} \sum_{j=1}^{\lfloor n / i\rfloor}(n-i j)$, which is consistent with the formula of $N_{d}$ from the second equality in the proof of Theorem 3 .

\section{The Algorithm and Some Applications}

In this section, our aim is to give an algorithm to compute the multiplicity of a proportionally modular numerical 
OUTPUT: $m(S(a, b, c))$.

INSTRUCTIONS:

(1) $a:=a \bmod b$.

(2) If $a>(b+c) / 2$, then $a:=b+c-a$.

(3) If $a \leq c$, then return 1 .

(4) If $a \mid b$, then return $b / a$.

(5) $k:=1$.

(6) while $(-k b) \bmod a>k b c /(a-c)$ do $k:=k+1$.

(7) return $\lceil k b / a\rceil$.

Algorithm 1: INPUT: positive integers $a, b$, and $c$.

semigroup and apply this algorithm to solve some questions on numerical semigroups.

Lemma 6 (Proposition 2 (2) in [6]). If $a \mid b$, then $m(a, b, c)=b / a$.

Remark 5. The observations made at the beginning of Section 2 and the fact $m(\mathbb{N})=1$ justify the steps (1), (2), and (3). Step (4) is a consequence of Lemma 6. Finally, steps (5), (6), and (7) are consequences of Corollary 1. Also by Corollary 1, we know that the Algorithm 1 stops after a finite number of steps.

At last, we apply the given algorithm to three questions on numerical semigroups. Given two relatively prime positive integers $a_{1}$ and $a_{2}$, we know from Lemma 18 in [5] that $\left\langle a_{1}, a_{2}\right\rangle=\left\{x \in \mathbb{N} \mid a_{2}^{-1} a_{2} x \bmod a_{1} a_{2} \leq x\right\}$, where $a_{2}^{-1} \in\left\{i \in \mathbb{N} \mid 0 \leq i<a_{1}, i a_{2} \equiv 1\left(\bmod a_{1}\right)\right\}$. Given two relatively prime positive integers $a_{1}$ and $a_{2}$ and a positive integer $d$, it is an open problem (see [1]) to find a formula for the smallest multiple of $d$ that belongs to $\left\langle a_{1}, a_{2}\right\rangle$.

Remark 6. Consider the quotient $\left\langle a_{1}, a_{2}\right\rangle / d=\{x \in$ $\left.\mathbb{N} \mid \mathrm{d} x \in\left\langle a_{1}, a_{2}\right\rangle\right\}=\left\{x \in \mathbb{N} \mid a_{2}^{-1} a_{2} \mathrm{~d} x \bmod a_{1} a_{2} \leq \mathrm{d} x\right\} \quad$ whose multiplicity is $m\left(\left\langle a_{1}, a_{2}\right\rangle / d\right)=m\left(S\left(a_{2}^{-1} a_{2} d, a_{1} a_{2}, d\right)\right)$, and therefore it can be calculated by applying Algorithm 1 .

Given a nonempty subset $A$ of $\mathbb{Q}_{0}^{+}$(here, $\mathbb{Q}_{0}^{+}$is the set of nonnegative rational numbers), we denote by $\langle A\rangle$ the submonoid of $\left(\mathbb{Q}_{0}^{+},+\right)$generated by $A$, that is, $\langle A\rangle=\left\{\lambda_{1} a_{1}+\cdots+\lambda_{n} a_{n} \mid n \in \mathbb{N}_{+}, \lambda_{1}, \ldots, \lambda_{n}\right.$

$\in \mathbb{N}$ and $\left.a_{1}, \ldots, a_{n} \in A\right\}$. Clearly, $S(A)=\langle A\rangle \cap \mathbb{N}$ is a submonoid of $\mathbb{N}$. Let $p$ and $q$ be two positive rational numbers with $p<q$. We use the notations $[p, q]=\{x \in \mathbb{Q} \mid p \leq x \leq q\}$ and $] p, q[=\{x \in \mathbb{Q} \mid p<x<q\}$. It is known that $S([p, q])$ and $S(] p, q[)$ are proportionally modular numerical semigroups. If $S$ is a numerical semigroup, then the greatest integer that does not belong to $S$ is called the Frobenius number of $S$ and denoted here by $F(S)$. In [9], a relationship between $F(S(a, b, 1))$ and $m(S](b / a), b /(a-1)[)$ is given by the following three lemmas.

Lemma 7 (Proposition 1 in [9])

(1) $S([b / a, b /(a-c)])=S(a, b, c)$
(2) Conversely, if $a_{1}, b_{1}, a_{2}$, and $b_{2}$ are positive integers $\begin{array}{ll}\text { such that } \quad\left(b_{1} / a_{1}\right)<\left(b_{2} / a_{2}\right), & \text { then } \\ S\left(\left[\left(b_{1} / a_{1}\right),\left(b_{2} / a_{2}\right)\right]\right)=S\left(a_{1} b_{2}, b_{1} b_{2}, a_{1} b_{2}-a_{2} b_{1}\right)\end{array}$

Lemma 8 (Theorem 9 in [9]). $S(] b / a, b /(a-1)[)=$ $S\left(\left[\left(\left(2 b^{2}+1\right) / 2 a b\right),\left(\left(2 b^{2}-1\right) / 2 b(a-1)\right)\right]\right)$.

Lemma 9 (Theorem 18 in [9]). Let $S=S([b / a, b /(a-1)])$ and $T=S(] b / a, b /(a-1)[)$. Then, $F(S)=b-m(T)$.

By Lemma $7(1)$, we have $S(a, b, 1)=S(] b / a, b /(a-1)[)$. Moreover, Lemmas 8 and 9 assert that $S(] b / a, b /(a-1)])=S\left(4 a b^{3}-2 a b, 4 b^{4}-1,4 b^{3}-4 a b+2 b\right)$. Therefore, $\quad F(S(a, b, 1))=b-m\left(S\left(4 a b^{3}-2 a b\right.\right.$, $\left.\left.4 b^{4}-1,4 b^{3}-4 a b+2 b\right)\right)$. Next, we generalize the result to $S(a, b, c)$.

Theorem 4. Let $c \mid a$ and $c \mid b$. Then, $F(S(a, b, c))=(b / c)-$ $m\left(S\left(4 a b^{3}-2 a b c, 4 b^{4}-c^{2}, 4 b^{3} c-4 a b c+2 b c^{2}\right)\right)$.

Proof. Let $a=c a_{1}$ and $b=c b_{1}$. Then, $a x \bmod b \leq c x$ if and only if $a_{1} x \bmod b_{1} \leq x$. It follows that $S(a, b, c)=S\left(a_{1}, b_{1}, 1\right)=S((a / c),(b / c), 1) . \quad$ Therefore, $F(S(a, b, c))=(b / c)-m\left(S\left(4 a b^{3}-2 a b c, 4 b^{4}-\right.\right.$

$\left.\left.c^{2}, 4 b^{3} c-4 a b c+2 b c^{2}\right)\right)$.

Remark 7. From Theorem 4, we can apply Algorithm 1 to compute $F(S(a, b, c))$ if $c$ is a common divisor of $a$ and $b$.

Let $S$ be a numerical semigroup minimally generated by $\left\{n_{1}, n_{2}\right\}$. Sylvester proved in [10] that $F(S)=n_{1} n_{2}-n_{1}-n_{2}$. If $S$ is minimally generated by $\left\{n_{1}, n_{2}, \ldots, n_{p}\right\}$, with $p>2$, then it is still an open problem to find a formula for $F(S)$. Next, we apply our algorithm to compute the Frobenius number of a numerical semigroup minimally generated by $\left\{n_{1}, n_{2}, n_{3}\right\}$.

Let $n_{1}, n_{2}$, and $n_{3}$ be three positive integers such that $\operatorname{gcd}\left(n_{1}, n_{2}, n_{3}\right)=1$ and $n_{1}>n_{2}>n_{3}$, denoted by $S$ the numerical semigroup $S:=\left\langle n_{1}, n_{2}, n_{3}\right\rangle$. In the study of $F(S)$, we can assume that $\operatorname{gcd}\left(n_{i}, n_{j}\right)=1$, for $i \neq j$ with $i, j \in\{1,2,3\}$. This is due to the following result.

Lemma 10 (Theorem 3 in [11]). Let $n_{1}, n_{2}, n_{3} \in \mathbb{N}_{+}$and $d_{i j}=$ $d_{j i}=\operatorname{gcd}\left(n_{i}, n_{j}\right)$ for every $\{i, j\} \subset\{1,2,3\}$ with $i \neq j$. Define 
$m_{1}, m_{2}, m_{3} \in \mathbb{N}_{+}$such that $n_{i}=m_{i} d_{i j} d_{i k}$ for every $\{i, j, k\}=\{1,2,3\}$. Then,

$F\left(\left\langle n_{1}, n_{2}, n_{3}\right\rangle\right)=d_{12} d_{23} d_{31} F\left(\left\langle m_{1}, m_{2}, m_{3}\right\rangle\right)$.
Remark 8. Denote by $L_{i}$ the positive integer $\min \left\{x \in \mathbb{N}_{+} \mid x n_{i} \in\left\langle n_{j}, n_{k}\right\rangle\right\}$, with $\{i, j, k\}=\{1,2,3\}$. In Theorem 3.4 in [12], it is proved that

$$
F(S)=L_{1} n_{1}+\max \left\{\left(L_{2} n_{2} n_{3}^{-1} \bmod n_{1}\right) n_{3},\left(L_{3} n_{3} n_{2}^{-1} \bmod n_{1}\right) n_{2}\right\}-n_{1}-n_{2}-n_{3}
$$

Observe that, by applying Algorithm 1, we obtain $L_{1}, L_{2}$, and $L_{3}$. Thus, we have $F\left(\left\langle n_{1}, n_{2}, n_{3}\right\rangle\right)$ by the previous formula.

\section{Data Availability}

The data used to support this study are available upon request to the corresponding author.

\section{Conflicts of Interest}

The author declares that there are no conflicts of interest.

\section{Acknowledgments}

This work was supported by the National Natural Science Foundation of China (Nos. 11701504 and 11801081), the Characteristic Innovation Project of Department of Education of Guangdong Province (No. 2020KTSCX159), the Science and Technology Innovation Guidance Project of Zhaoqing City (No. 2021040315026), the Innovative Research Team Project of Zhaoqing University, and the Scientific Research Ability Enhancement Program for Excellent Young Teachers of Zhaoqing University.

\section{References}

[1] J. C. Rosales and P. A. García-Sánchez, Numerical Semigroups, Springer, New York, NY, USA, 2009.

[2] J. C. Rosales, P. A. Garća-Sánchez, J. I. García-Garćia, and J. M. Urbano-Blanco, "Proportionally modular Diophantine inequalities," Journal of Number Theory, vol. 103, no. 2, pp. 281-294, 2003.

[3] J. C. Rosales and P. A. García-Śanchez, "Numerical semigroups having a Toms decomposition," Canadian Mathematical Bulletin, vol. 51, no. 1, pp. 134-139, 2008.

[4] J. C. Rosales and J. M. Urbano-Blanco, "Proportionally modular Diophantine inequalities and full semigroups," Semigroup Forum, vol. 72, no. 3, pp. 362-374, 2006.

[5] J. C. Rosales and P. Vasco, "The smallest positive integer that is solution of a proportionally modular Diophantine inequality," Mathematical Inequalities and Applications, vol. 11, no. 2, pp. 203-212, 2008.

[6] A. Moscariello, "On the least positive solution to a proportionally modular Diophantine inequality," Integers, vol. 16, Article ID A34, 2016.

[7] M. Delgado and J. C. Rosales, "On the Frobenius number of a proportionally modular Diophantine inequality," Portugaliae Mathematica, vol. 63, no. 4, pp. 415-425, 2006.

[8] The On-Line Encyclopedia of Integer Sequences, https://www. oeis.org.
[9] J. C. Rosales and P. Vasco, "On the Frobenius number of a modular diophantine inequality," Mathematica Bohemica, vol. 133, no. 4, pp. 367-375, 2008.

[10] J. J. Sylvester, "Mathematical questions with their aolutions," Educational Series, vol. 41, no. 21, p. 6, 1884.

[11] S. M. Johnson, "A linear Diophantine problem," Canadian Journal of Mathematics, vol. 12, pp. 390-398, 1960.

[12] A. Moscariello, "The first elements of the quotient of a numerical semigroup by a positive integer," Semigroup Forum, vol. 90, no. 1, pp. 126-134, 2015. 